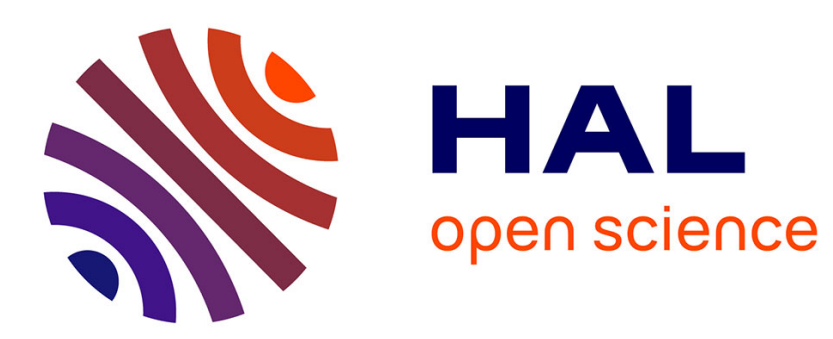

\title{
Le marché de l'aide sociale: représentations, paradoxes et enjeux
}

Franck Cochoy, Ygal Fijalkow

\section{To cite this version:}

Franck Cochoy, Ygal Fijalkow. Le marché de l'aide sociale: représentations, paradoxes et enjeux. Politiques et Management public, 2002, 20 (2), pp.117-141. hal-00189047

\section{HAL Id: hal-00189047 https://hal.science/hal-00189047}

Submitted on 19 Nov 2007

HAL is a multi-disciplinary open access archive for the deposit and dissemination of scientific research documents, whether they are published or not. The documents may come from teaching and research institutions in France or abroad, or from public or private research centers.
L'archive ouverte pluridisciplinaire HAL, est destinée au dépôt et à la diffusion de documents scientifiques de niveau recherche, publiés ou non, émanant des établissements d'enseignement et de recherche français ou étrangers, des laboratoires publics ou privés. 


\title{
Centre d'Étude et de Recherche Technique, Organisation, Pouvoir
}

\section{Le marché de l'aide sociale : représentations, paradoxes et enjeux}

\author{
Franck Cochoy \& Ygal Fijalkow
}

\begin{abstract}
Résumé. Le soutien indirect et décentralisé de l'État envers les associations et les initiatives locales d'aide sociale a entraîné une prolifération et une diversification considérables de l'offre de services sociaux. Quelle peut être l'incidence d'un tel constat? Les résultats d'un questionnaire administré auprès des usagers de la Caisse Régionale d'Assurance Maladie montrent que règnent un certain nombre de confusions dans l'esprit du public quant aux prestations qui lui sont offertes. Nous ferons l'hypothèse que de telles confusions, loin de trouver leur seule origine dans l'ignorance ou dans l'incompétence des usagers, tiennent largement à la configuration particulière du secteur concerné. Une série d'entretiens conduits auprès de différents intervenants sociaux auprès des personnes âgées montre que les relations de coopération, de juxtaposition ou de concurrence entre ces agents éclairent les difficultés que rencontrent les usagers pour s'orienter entre ces différents prestataires qui, s'ils se présentent séparément comme autant de «services publics », offrent collectivement et malgré eux toutes les apparences d'un véritable «bazar » du social.
\end{abstract}

Mots clés : service public, relation de service, concurrence, personnes âgées.

\begin{abstract}
In France, State support for social NGOs and decentralized social programs led to a considerable diversification of the supply of social services. What are the consequences of such a statement ? We will try to answer this question through a case study of social services for elderly people. The results of a survey of CRAM's users (CRAM: the French national pension fund) will first show that the public has a very confused view of the services provided to them. Our hypothesis is that this problem does not find its explanation in the public's ignorance, but rather in the particular configuration of the supply side of social services. In order to back such a hypothesis, we will go beyond the case of the CRAM. Through interviews conducted with different providers of social services for elderly people, we will show that the particular relationships between these actors (cooperation, juxtaposition, competition...) explain the difficulty of elderly people to find their way among these institutions and actors that present themselves as a true "social bazaar".
\end{abstract}

Key words: public services, service relationship, competition, elderly people.

Franck Cochoy : cochoy@univ-tlse2.fr ; Ygal Fijalkow : ygal@univ-tlse2.fr 

Depuis quelques années, un nombre considérable d'études ont été consacrées à l'action publique, aux relations de service, aux rapports entre l'administration et ses usagers (Weller, 1998 [38]). L'émergence de ces études doit beaucoup à la conjonction de trois phénomènes : la montée de la précarité et de l'exclusion au terme de trente années de crise (Fitoussi \& Rosanvallon, 1996 [17] ; Paugam, 1996 [30]), les difficultés de l'État providence, qui ne peut plus tout prendre en charge dans un contexte d'assainissement et de restriction des dépenses publiques (Rosanvallon, 1998 [33]), et la nécessité d'un renouveau de l'action publique, notamment sous la double pression des problèmes sociaux et (dans une moindre mesure ${ }^{1}$ ) des mouvements citoyens (Piotet, 1997 [31] ; Fijalkow, 2002 [16]). Dans ce contexte, l'analyse des relations de service s'est efforcée de montrer comment le soutien des initiatives locales, la meilleure prise en compte de l'usager ${ }^{2}$ et la mise en place d'un «pilotage par le bas » (ou modernisation des administrations «par l'usager» [Strobel, 1993] [34]) des administrations permettent à l'État de se faire à la fois plus modeste et plus performant, notamment pour la prise en charge des plus démunis ${ }^{3}$.

Pourtant, nous voudrions attirer l'attention sur des effets paradoxaux et jusque-là peu pris en compte de cette dernière politique, des effets qui pèsent pourtant lourdement sur les résultats

1. On gagnerait peut-être à distinguer pression directe et indirecte du public : si nous rejoignons Michel Chauvière (1997 [6]) lorsqu'il souligne que les mobilisations d'usagers constituent des effets correctifs qui agissent le plus souvent à la marge et sans grand pouvoir de décision sur les cibles vi sées, nous pensons néanmoins que les mouvements de grève, l'invocation rhétorique de la figure du client (Jeannot, 1999 [23]) et la montée en compétence des citoyens-consommateurs (Cochoy, 2001a [7]) ont sans nul doute contribué à créer (ou tout au moins à hâter), dans l'esprit des responsables politiques et administratifs, le souci d'une amélioration réelle des relations entre les administrations et leurs usagers.

2. D'où le succès de la notion de «coproduction» du service (Gadrey, 1994 [18]), qui vise à mettre l'accent sur le caractère non plus unilatéral mais bilatéral de la prestation, et donc sur la nécessité d'associer chaque administré à la production du service qui lui est rendu. On a beaucoup loué les vertus humaines et fonctionnelles de cette façon de voir, mais on a peu vu ses effets pervers. Or l'idée de coproduction amène parfois à faire partager à l'usager la responsabilité de la déviation du service auquel il pouvait s'attendre (par exemple, lorsque l'ANPE redéfinit ses missions en termes d'accompagnement individualisé des personnes indépendamment de la mise à disposition réelle d'un emploi, elle enrôle le chômeur coproducteur du service dans l'acceptation tacite au pire d'une non-prestation, au mieux d'une prestation de substitution. Sur ce point, cf. Cochoy \& de Terssac, 1999 [12]).

3. L'analyse des relations de service s'est aussi efforcée de montrer les décalages qui se font jour au sein même de l'administration entre le projet des états-majors administratifs et les capacités réelles d'action des administrations déconcentrées (Warin, 2000 [37]). En effet, la relation de service engage bien plus que l'efficacité managériale (Warin, 1999 [36]) : elle met notamment en jeu une compétence relationnelle des agents qui se construit dans l'interaction avec l'usager, et qui échappe donc à la logique des dispositifs de gestion préalables et standardisés (Borzeix, 1995 [2]), de sorte que la multiplication des innovations dans le service public se traduit bien souvent par un problème de stabilité et un déficit de coordination (Weller, 1999 [39]). 
recherchés : le soutien indirect et décentralisé des associations et des initiatives locales, le souci de mieux prendre en compte les missions de service public et la volonté de répondre plus précisément aux attentes des usagers ont entraîné une prolifération et une diversification considérables de l'offre de services sociaux. Partant, on observe le développement croissant, plus ou moins visible et non désiré comme tel, d'un véritable « marché de l'aide sociale »4.

Ce marché combine, associe ou juxtapose les actions conjuguées des administrations centrales et locales, des associations locales et/ou caritatives, des organismes privés (etc.), de sorte que la complexité apparaît comme une des caractéristiques majeures du secteur de l'action sociale (Chauvière, 2000 [6]). La question qui se pose est alors celle de l'adaptation de cette offre multiple aux publics auxquels elle s'adresse : n'est-il pas paradoxal d'aider des gens que l'on peut considérer en première approximation comme des victimes du marché (perte d'emploi, manque de ressources, incapacité à «tenir» seul dans un univers individualiste et compétitif... $)^{5}$ en présentant les moyens d'en sortir sous la forme d'un véritable marché ? S'orienter dans le marché de l'aide sociale nécessite de trouver la «bonne porte», de maîtriser l' «information », de planifier ses déplacements et ses rendez-vous. C'est beaucoup

4. L'usage de l'analogie marchande pour un tel cas peut surprendre, si l'on entend par marché un espace organisé censé mettre en relation des agents calculateurs, étrangers les uns aux autres à l'entrée comme à la sortie (Callon, 1998 [3]), un espace dont les acteurs sont à la fois mus par leur seul intérêt individuel et concurrents entre eux, un espace où les ajustements, monétaires, se font uniquement sur les prix, conformément aux traits idéal-typiques du «monde marchand» (Boltanski \& Thévenot, 1991 [1]). Pourtant, deux raisons opposées nous semblent justifier l'emploi de cette analogie. D'abord, il convient de noter que la médiation des prix ou l'anonymat réciproque des acteurs ne peuvent plus servir à caractériser le fonctionnement des marchés d'aujourd'hui : les acteurs des marchés modernes tentent de surmonter les contraintes d'une concurrence tarifaire aveugle en jouant sur la diversification qualitative de leurs prestations (Karpik, 1989 [26] ; Callon \& al. 2001 [4] ; Cochoy, 2002 [10]) et sur la fidélisation d'une clientèle certes souvent inconnue au départ, mais avec laquelle ils souhaitent nouer, à l'issue des transactions, un rapport d'interconnaissance stable et si possible exclusif (Cochoy, 2001c [9]). Or comme nous le verrons, dans le secteur de l'aide aux plus démunis, on observe bien l'existence d'un public pluriel, souvent mal connu mais que les acteurs de l'offre tentent justement de saisir, de connaître, voire de fidéliser, via l'engagement de prestations concurrentes et différenciées. Ensuite et a contrario, le renouvellement et la sophistication de la donne marchande ne font nullement obstacle à la permanence d'un certain nombre de ses caractéristiques classiques : multiplicité et diversité de l'offre — donc «embarras du choix » —, hétérogénéité et mobilité des publics, existence d'intérêts propres aux acteurs, autant de traits dont nous verrons qu'ils se retrouvent aussi dans le secteur de l'aide sociale, sans bien sûr empêcher l'expression d'autres attitudes comme l'altruisme, l'empathie ou la coopération. La véritable limite à l'usage de l'analogie marchande concerne plutôt, selon nous, la conception des «publics » en termes de « demande » et d' " agents calculateurs ». Ce point crucial est l'objet même de notre analyse d'ensemble ; nous y reviendrons donc « après examen », en conclusion.

5. On retrouve ici la conception introduite par Karl Polanyi (1983 [32]), qui a montré comment l'avènement du marché du travail, en «coupant» les personnes de leur communauté d'appartenance afin de permettre leur libre circulation sur le marché de l'emploi, s'est accompagné de toute une série de maux sociaux imputables à la disparition des cadres protecteurs. 
demander à des personnes en situation précaire, dont l'horizon temporel s'est brouillé, et dont les capacités sociales et cognitives sont fragilisées. Il nous apparaît donc important de rendre visible ce «marché de l'aide sociale», ses aspects et ses enjeux, afin de poser sinon la question de sa pertinence, du moins celle de sa maitrise.

Pour ce faire, nous nous appuierons sur un travail de terrain mené auprès des milieux de l'aide aux personnes âgées ${ }^{6}$. Nous partirons du cas de la Caisse Régionale d'Assurance Maladie - l'un des acteurs centraux de ce secteur. La présentation des résultats d'un questionnaire administré auprès des usagers de cet organisme chargé du versement des retraites — comme son nom ne l'indique pas ! - nous permettra de mettre à jour un certain nombre de confusions qui règnent dans l'esprit du public quant aux prestations qui lui sont offertes. Nous ferons l'hypothèse que de telles confusions, loin de trouver leur seule origine dans l'ignorance ou dans l'incompétence des usagers, tiennent largement à la configuration particulière du secteur concerné. Pour corroborer cette hypothèse, nous élargirons notre étude au-delà de la CRAM. En nous appuyant sur une série d'entretiens menés auprès des différents acteurs censés fournir une aide sociale aux personnes âgées (institutions d'aide sociale, associations humanitaires, maison de retraites...), nous montrerons comment le positionnement de chacun de ces intervenants, mais aussi leurs relations de coopération, de juxtaposition ou de concurrence, permettent de rendre compte des difficultés que rencontrent les usagers pour s'orienter entre ces différents prestataires qui, s'ils se présentent chacun comme autant de « services publics », offrent collectivement et malgré eux toutes les apparences d'un véritable « bazar » du social.

\section{Service public et services perçus : ce que nous apprend le public de la CRAM}

L'étude des relations de service et de l'interaction entre agents et usagers intervient généralement en aval des démarches individuelles, lorsque la personne a déjà «passé la porte » de l'organisme recherché. Ce cadrage particulier de la scène d'observation peut

6. L'enquête a été réalisée en 1999-2000 dans le cadre d'une «étude-école » de l'IUP de sociologie appliquée de l'Université Toulouse II, encadrée par les auteurs et Sandrine Barrey, avec le concours de l'ensemble des étudiants de deuxième année. Qu'ils en soient vivement remerciés. On trouvera une présentation des résultats d'ensemble dans le rapport correspondant (Cochoy, 2001b [8]). Une version antérieure du présent article a fait l'objet d'une communication dans le cadre de la $8^{\text {ème }}$ journée d'étude du CLERSÉ, L'organisation sociale de l'économie, Villeneuve d'Ascq, vendredi 8 juin 2001. Nous remercions les participants de ce colloque, Christophe Beslay, ainsi que les lecteurs de PMP pour nous avoir aidés, par leurs remarques, à améliorer notre texte. Bien entendu les propos qui sont tenus ici n'engagent que nous. 
conduire le chercheur à laisser de côté la question de l'identification même de la «bonne porte » et du service recherché, et à concentrer son étude sur l'ajustement problématique qui s'établit entre un public et un prestataire supposés partager une connaissance commune et minimale de la prestation. Pourtant, même lorsqu'on s'intéresse à des usagers «en situation », dans l'enceinte même de l'organisme prestataire, on s'aperçoit que leur représentation de ce qu'ils cherchent ou de ce qu' on peut leur offrir est particulièrement confuse. C'est ce constat qui ne laisse pas d'avoir des conséquences sur la délivrance des prestations — que nous voudrions mettre en évidence, à partir d'une enquête par questionnaire menée auprès des usagers de la CRAM, lors de leur visite à cet organisme ${ }^{7}$.

\section{A. Le statut de la CRAM}

Avant d'étudier la représentation des prestations proprement dites, nous avons dans un premier temps cherché à savoir comment les usagers situaient la CRAM dans l'espace des intervenants potentiels. Pour ce faire, nous leur avons demandé de nous indiquer le statut de la CRAM parmi quatre choix possibles, dont un seul était exact. Il apparaît que les personnes identifient la CRAM comme un service public à $75 \%$ (voir graphique ci-dessous), alors que la réponse correcte (soit un établissement public géré par des fonds privés) ne recueille que $15,3 \%$ de suffrages. En gros cependant, les réponses sont logiques et pertinentes : si la solution correcte est repoussée, c'est sans doute parce qu'elle comporte le terme «privé » — un terme qui, lorsqu'il est présenté seul, ne recueille presque aucun avis $(0,3 \%)$. De même, si la solution «association municipale» $(0,8 \%)$ ne retient l'attention de presque personne, c'est probablement parce que, dans l'esprit des gens, aucun lien logique n'émerge entre «retraite » et «mairie». Ces résultats montrent clairement que les usagers catégorisent les organismes prestataires de façon subjective et déductive, d'après leur mission, leur «secteur d'intervention », et non d'après une information préalable sur le fondement juridique des institutions. Mais un tel résultat permet aussi de s’interroger sur la vulnérabilité potentielle des personnes vis-à-vis de tout intervenant qui se présenterait à elles sous le jour du «service

7. Le questionnaire a été administré en février-mars 2000 auprès de 360 usagers de la CRAM MidiPyrénées, dans les locaux même de l'institution. Le fait que les usagers aient une représentation confuse des prestations que leur délivre un organisme alors même qu'ils s'y sont rendus et qu'on les interroge dans le cadre de leur visite permet de supposer que règne une confusion plus grande encore du côté de tous les bénéficiaires que nous aurions pu interroger chez eux sur les prestations délivrées par le même organisme. 
rendu » et de l' " aide due au public », un peu comme le loup trempe sa patte dans la farine pour tromper les petits chevreaux. Nous y reviendrons.

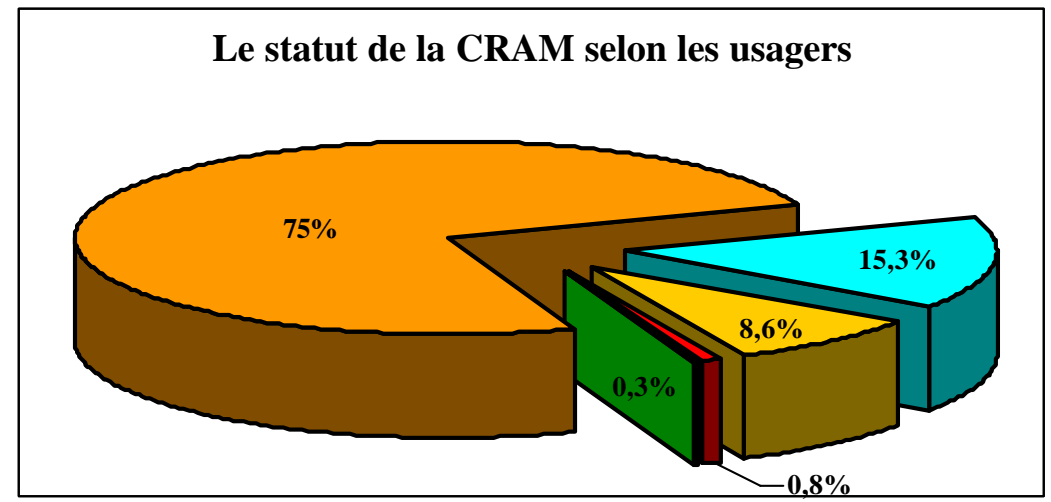

$75 \%$ : service public ; $15,3 \%$ : établissement géré par des fonds privés mais à but public ;

$8,6 \%$ ne sait pas ; $0,8 \%$ : association municipale ; $0,3 \%$ : entreprise privée

Si l'on entre maintenant dans le détails des prestations, on peut s'interroger sur l'aptitude des usagers à identifier les services offerts par l'organisme auquel ils s'adressent. Cette question peut sembler inutile si l'on souscrit au syllogisme raisonnable censé gouverner le service public concerné : si la CRAM verse les retraites et si les usagers se rendent à la CRAM, c'est évidemment pour régler avec la CRAM leurs affaires de retraite ! ${ }^{8}$ Pourtant, comme nombre de services publics, la CRAM dispense bien d'autres prestations que le simple versement des retraites (aide à domicile, allocations pour financer des voyages, etc.). D'où les deux questions suivantes : les usagers ont-ils connaissances des prestations «additionnelles » de la CRAM ? Pour une prestation donnée, les usagers s'orienteraient-ils prioritairement vers cet organisme ?

\section{B. Les services de la CRAM}

Afin d'évaluer la connaissance que les usagers ont des services proposés par la CRAM, nous leur avons proposé de nous dire quels services étaient à leur avis assurés par l'institution,

8. Ce syllogisme n'est pas dénué de fondement : $94 \%$ des personnes interrogées pensent que le rôle de la CRAM est de verser des retraites. Cependant, les personnes venues pour des demandes administratives (calculer leur retraite, faire un relevé de carrière, constituer ou suivre leur dossier) ne limitent pas le rôle de la CRAM à la gestion des retraites; de façon générale d'ailleurs, près de $77 \%$ de la population interrogée pensent que la CRAM doit accompagner les retraités tout au long de leur vie et environ $55 \%$ considèrent qu'elle doit fournir une aide sociale. 
en les invitant à choisir ces services parmi une liste d'affirmations dont certaines étaient vraies et d'autres fausses. Du côté des affirmations exactes, nous avions l'aide ménagère, l'aide à domicile, les subventions à un club sportif, les voyages... autant de services réellement proposés par l'institution'. Du côté des affirmations erronées nous avions l'aide alimentaire, l'accès aux maisons de retraite et l'aide médicale, qui sont autant de prestations non fournies par la CRAM.

Nous pouvons en premier lieu constater, qu'il s'agisse de questions vraies ou fausses, que le taux des personnes interrogées répondant «ne sait pas » est très élevé. Selon les items, ce taux varie entre $43,9 \%$ et $61,4 \%$. La difficulté de lecture des prestations offertes dans un secteur où les offres de service prolifèrent apparaît nettement : dès lors qu'on aborde les prestations qui sortent des missions prioritaires d'un organisme, les personnes ont bien du mal à savoir qui fait quoi, à établir une correspondance entre services et institutions. En ce qui concerne la CRAM tout au moins, il est clair que les usagers témoignent d'une importante méconnaissance des services offerts par ce prestataire.

Nous avons choisi d'analyser ces différents résultats en classant les questions selon le pourcentage de réponses positives qu'elles ont respectivement recueilli (voir graphique cidessous) :

9. La CRAM nous a signalé, après la présentation de nos résultats, que la subvention accordée à un club sportif n'était plus de mise. Cette fluctuation dans les activités de la CRAM renforce notre thèse quant à la difficulté qu'il y a à distinguer le vrai du faux, pour le chercheur comme pour les usagers ! 


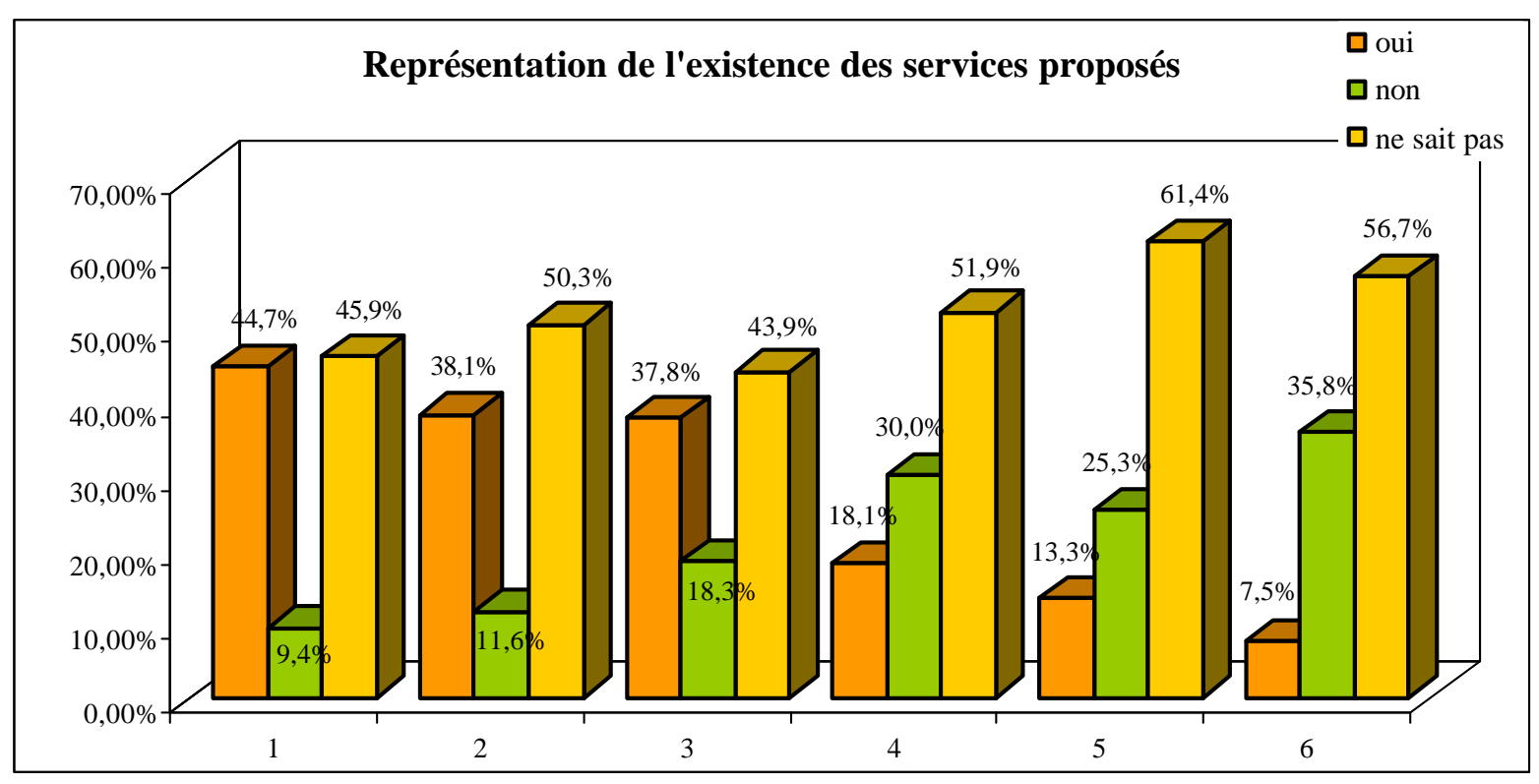

1 : accès aux maisons de retraite ; 2 : aide ménagère ; aide à domicile ; 3 : aide médicale ; 4 : voyages ; 5 : aide alimentaire ; 6 : subvention à des clubs sportifs

Il est à remarquer que la question qui obtient le plus de réponses affirmatives $(44,7 \%)$ est celle de l'accès aux maisons de retraites, alors que cette proposition est fausse ! On retrouve ici l'expression d'un processus cognitif évoqué plus haut, à savoir que les usagers procèdent avant tout par inférence logique subjective, plutôt que par information objective : de même que les usagers décrivent la CRAM comme «service public » en raison du lien qu'ils établissent entre l'institution et sa mission, ils attribuent au même organisme les prestations qui font intervenir son activité centrale, à savoir la question des « retraites ».

L'aide ménagère et l'aide à domicile, services effectivement proposés par la CRAM, sont identifiées comme relevant des missions de l'institution par 38,1\% des enquêtés. Ce point valorise la démarche de la CRAM qui a fait de ce service une priorité. Cependant, il convient de remarquer que 11,6\% des usagers nient l'existence de ce service, et que 50,3\% déclarent ne pas savoir s'il existe. Alors que l'aide alimentaire n'est pas une prestation fournie par la CRAM, 13,3\% des personnes interrogées croient tout de même que ce service est offert; $25,3 \%$ déclarent que non, tandis que $61,4 \%$ ne savent pas. Enfin, les questions des subventions à un club sportif et des voyages regroupent les plus forts taux de réponses négatives : respectivement $35,8 \%$ et $30 \%$. Ces deux propositions, qui sont (ou ont été) pourtant vraies, apparaissent fortement incongrues aux yeux des usagers. Il ressort de cet ensemble de résultats que dans l'esprit du public, la CRAM est principalement un organisme de versement des retraites, éventuellement fournisseur d'une aide sociale dont le sens reste flou. 
La méconnaissance massive des usagers quant aux services qui leur sont pourtant offerts laisse à penser que l'attribution des prestations méconnues risque d'être sélective et arbitraire, de sorte que l'on peut s'interroger sur la pertinence de cette diversification «généreuse » des services. Sur un véritable marché chaque professionnel de l'offre a souvent intérêt, plutôt qu'à multiplier les services, à se replier sur son «métier de base » et à afficher sa différence, selon la logique économique classique des «avantages comparatifs ». Or le marché de l'aide sociale se caractériserait plutôt par le mouvement inverse : sur ce marché, le principe d'action étant non pas la spécificité et la performance, mais la générosité et la reconnaissance, les acteurs institutionnels tentent d'offrir toujours plus, d'embrasser les pratiques des autres, sans bien sûr en faire forcément une stratégie délibérée ${ }^{10}$. Mais ce faisant, la bonne volonté des acteurs (et surtout la superposition non coordonnée des bonnes volontés) finit par brouiller la lisibilité de l'offre, au risque de laisser les populations les plus fragiles dans l'ignorance — alors que les offres additionnelles leur sont pourtant destinées.

\section{La bonne (ou la mauvaise ?) porte pour l'aide à domicile}

Une question est de savoir si les usagers connaissent les prestations offertes par la CRAM, une autre est de savoir si les mêmes usagers identifient bien les organismes susceptibles de leur apporter telle prestation. Nous avons essayé de répondre à cette dernière interrogation en posant aux usagers la question suivante : «si vous aviez besoin d'une aide à domicile, à qui vous adresseriez-vous en priorité ? ». Sept modalités de réponse étaient proposées aux usagers : la mairie, une association, l'entourage, la famille, la CRAM, la CPAM, et « autre».

10 Le cas de la CRAM en matière de prolifération des prestations n'a rien d'isolé. Prenons l'exemple de la $\mathrm{CAF}$, qui a l'avantage d'intervenir à l'autre extrême du cycle de vie, en s'occupant de façon prioritaire des enfants et de la jeunesse. La symétrie qui se fait jour entre le positionnement des deux organismes mérite d'être notée : de même que la CRAM ne s'occupe pas uniquement des retraites, la CAF ne se contente pas de l'aide aux familles. Au-delà du versement des prestations liées à l'enfance, comme les allocations familiales ou la compensation financière à l'interruption d'activité pour jeune enfant, la CAF accorde aussi des avantages dans le cadre de la retraite, dont le financement de l'assurance. La CAF assure la surveillance médicale gratuite du jeune enfant et de sa mère et des lieux d'accueil collectif des jeunes, elle subordonne l'octroi de certaines prestations aux examens médicaux obligatoires, elle délivre des prestations financières pour les locataires et accédants à la propriété, elle dispense des conseils, assure l'hébergement temporaire de familles défavorisées, cofinance des foyers de jeunes travailleurs, soutient financièrement les familles et les étudiants, intervient en matière d'accompagnement scolaire et finance des équipements de loisir. Un tel inventaire «à la Prévert » des missions de la CAF illustre bien la prolifération des champs d'intervention des organismes sociaux, et les superpositions récurrentes qui peuvent apparaître entre les «offres » des différents organismes : combien de personnes savent, en dehors du milieu de l'aide sociale (et même peut-être à l'intérieur de ce milieu ?) que la CAF participe à l'assurance retraite ou finance des équipements de loisir? 
L'aide à domicile est la principale prestation sociale soutenue par la CRAM. Or, pour obtenir ce type d'aide, 43,3\% des usagers s'adresseraient en priorité à la mairie, et 16,1\% à leur famille (voir graphique ci-dessous). Ce résultat illustre bien l'incertitude caractéristique de l'offre proliférante et non coordonnée de services sociaux : les prestations sont diverses, les acteurs multiples, chaque institution offre des services qui vont au-delà de ses missions strictes, et il résulte de ce foisonnement de bonnes volontés et d'initiatives une difficulté manifeste, pour les usager, à « lire » l'offre, à rapporter chaque prestation possible à un intervenant clairement identifié $^{11}$. Pour l'aide sociale à domicile, la CRAM n'intervient qu'en troisième position (devant les associations, la CPAM et l'entourage) avec 12,2\% des personnes, alors même, soulignons-le, que ces personnes sont des usagers de la CRAM, de surcroît interrogés dans l'enceinte même de l'institution qu'ils devraient connaître !

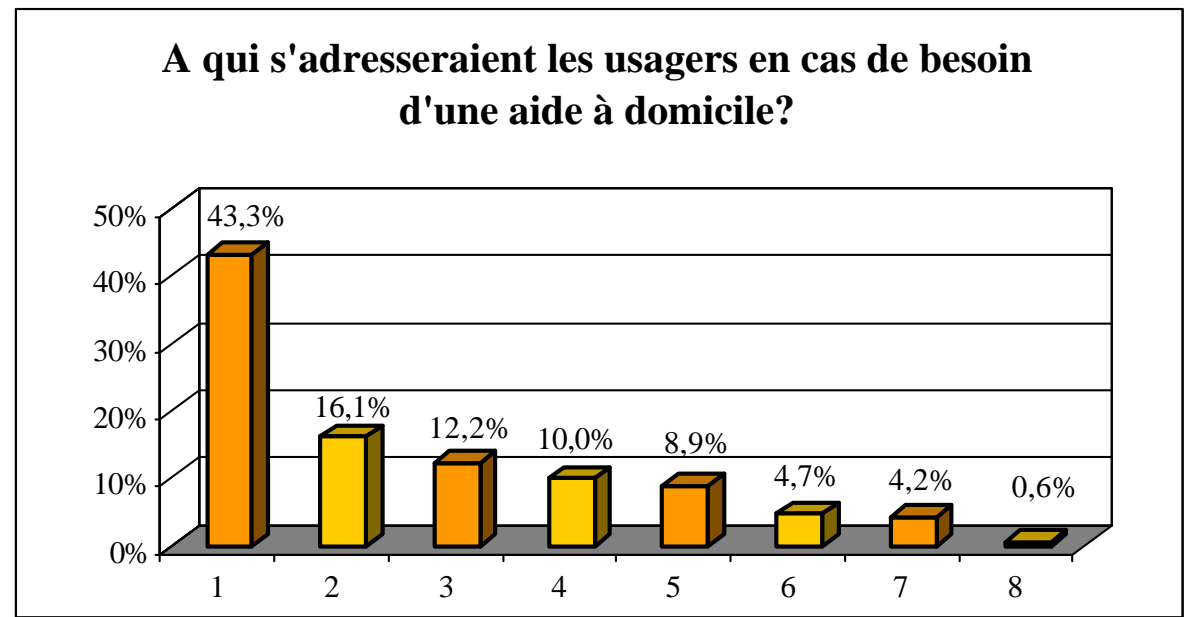

1 : la mairie ; 2 : la famille ; 3 : la CRAM ; 4 : les associations ;

5 : la CPAM ; 6 : l’entourage ; 7 : autre ; 8 : non réponse.

11. Une recherche de Frédéric Lesemann et Claire Chaume (1992 [27]) menée sur le rôle des familles dans le maintien à domicile de proches âgés fortement dépendants anticipait nos propres résultats : comme nous, les auteurs observaient qu'il existe un décalage considérable entre les représentations globales que se font les familles de l'offre de service et la spécificité des services offerts. En revanche, notre propre travail ne nous permet pas de rejoindre l'interprétation des auteurs qui discernaient derrière le constat empirique d'ignorance des usagers un intérêt stratégique à se laisser porter et assister par la bienveillance sociale des organismes prestataires. Bien au contraire, tous les agents que nous avons rencontrés ont insisté sur l'extrême rareté des comportements opportunistes de la part des usagers. Par ailleurs, d'un point de vue théorique, on peut douter que des personnes fragiles, isolées, peu informées, puissent faire preuve de calcul et de machiavélisme : il nous semble y avoir derrière une telle hypothèse une contradiction dans les termes. 


\section{Au-delà de la CRAM, les paradoxes du marché de l'aide sociale}

Que penser de ces premiers résultats ? D’un côté, la définition «large » des missions et le fait que chaque organisme fasse «plus », en réalité, que ce que l'on pourrait attendre de lui, peut aller dans le sens d'une meilleure prise en charge des situations difficiles. Si telle situation personnelle peut être prise en compte par plusieurs organismes, le secteur social dans son ensemble se donne plus de chances de «capter » les publics en difficulté, et donc de leur venir en aide. De même, l'anticipation par le public, même faible, de cette relative polyvalence ou pluri-activité des services sociaux peut amener les personnes à taper à n'importe quelle porte pour que s'enclenche un début de réponse à leurs problèmes, ne serait-ce que parce que la polyvalence et la pluri-activité institutionnelle confèrent aux personnels des institutions une connaissance des autres personnels et institutions vers lesquels il est possible d'aiguiller les usagers.

Mais d'un autre côté, la prolifération des services, le recouvrement des sphères de compétences et l'impossibilité pour le public de connaître l'ensemble de l'offre et sa répartition créent une confusion très préjudiciable à la bonne orientation des personnes, et à la gestion la meilleure et la plus rapide de leurs difficultés. La réalité d'un «marché de l'aide sociale » à entrées multiples crée l'illusion d'une situation de choix, ajoute à la recherche prioritaire d'une solution pour un problème personnel la recherche superflue de «la bonne porte », et finit donc par compliquer une situation que les institutions sont pourtant censées simplifier. Ce dilemme central s'accompagne de certains paradoxes que nous mettrons à jour en nous appuyant sur l'étude non plus de la «demande» (les usagers) mais de l' «offre» de services (au travers d'une série d'entretiens recueillis auprès des divers intervenants) ${ }^{12}$.

12. Plus précisément nous avons interrogé des représentants des grandes institutions d'aide sociale (Caisse Primaire d'Assurance Maladie, Caisse d'Allocations Familiales, Conseil Général de la Haute-Garonne, Centre Communal d'Action Sociale de la ville de Toulouse), des associations rattachées au CCAS (service de portage de repas à domicile, point info séniors, pôles troisième âge de quartier...), des associations caritatives, des responsables de trois maisons de retraites, un écrivain public, un prêtre... Il s'est agi non pas de faire le tour exhaustif des prestataires potentiels, mais de recueillir l'avis d'intervenants diversifiés, de façon à obtenir un premier aperçu des différentes facettes du «marché de l'aide sociale ». 


\section{A. Le paradoxe d'un double problème social, ou la nécessaire « aide à l'aide »}

La notion de «problème social », que l'on réfère souvent à tort à la dimension unique du problème personnel, de la «situation», du «cas » particuliers (même si bien sûr tout cas particulier peut en lui-même être extrêmement polymorphe et multidimensionnel), revêt la plupart du temps une double dimension. La première dimension est celle, personnelle, qui tient à la difficulté ou aux difficultés propres à l'usager. Mais la seconde dimension est celle, plus collective mais paradoxalement moins connue et tout aussi importante, qui relève de la configuration institutionnelle du réseau d'aide aux personnes. Cette dernière dimension, en venant rejoindre la première, aboutit au paradoxe suivant : le réseau d'aide sociale, censé en principe résoudre les problèmes personnels, ajoute pourtant dans un premier temps un problème supplémentaire à ceux qu'il est censé $\operatorname{traiter}^{13}$ : non seulement les personnes éprouvent des difficultés et doivent connaître leurs droits, mais pour résoudre ces difficultés et accéder à cette première connaissance, il leur faut encore connaître ceux qui peuvent les aider à surmonter leurs difficultés et à les faire «entrer» dans leurs droits, c'est-à-dire trouver le moyen — et le plus souvent la chance — d'entrer en contact avec les bons prescripteurs :

"C'est vrai qu'il y aurait certainement une tranche de population qui aurait plus besoin de nous et qui ne nous connaît pas, on se le demande parce que... (souffle) on peut pas... c'est pas la majorité des personnes qui sont vraiment tellement isolées, sans amis, sans voisinage, sans rien du tout. »(Association humanitaire).

Force est de constater l'extrême difficulté qu'il y a à connaître le marché de l'aide sociale, tant en matière de prestations (droits) que de prestataires (services censés mettre les personnes face à leurs droits). Une incursion rapide dans ce secteur montre qu'il se caractérise à la fois par un dévouement et par une sollicitude ${ }^{14}$ indiscutables de l'ensemble des acteurs ${ }^{15}$ et par un

13. On retrouve ici les enseignements des études de la cognition située, et notamment des travaux de Donald Norman (1993 [29]), qui montre que tout artefact cognitif censé améliorer les performances des acteurs «du point de vue du système » ajoute dans un premier temps une difficulté d'adaptation à l'artefact luimême « du point de vue de l'acteur ».

14. Anni Borzeix (1995 [2]) a souligné la bienveillance assez générale que les agents manifestent à l'égard des étrangers : respect, courtoisie, tolérance, sollicitude et réceptivité. Pour elle, cette bienveillance est un procédé compensatoire consistant pour les agents concernés à réparer, par des gestes, des répétitions et des reformulations, le préjudice subi par les étrangers du fait de leur moindre accès aux ressources cognitives, culturelles et langagières dont bénéficie habituellement l'usager moyen. De façon analogue, Jacques Gautrat (1996 [20]) a montré au travers d'une étude menée pendant cinq ans sur les CAF de Foix, Quimper, Tours et Tarbes auprès des populations les plus démunies, que les agents se référent à un registre de justice identique à celui des travailleurs sociaux et des bénévoles plutôt qu'à une logique bureaucratique où ils n'auraient pas à se 
règne non moins patent de l'arbitraire, de la chance ou du hasard des « bonnes » rencontres (ou des démarches inadéquates). Qui connaît, par exemple, l'action du «Point info senior» de Toulouse $?^{16}$ Cette structure, encore très récente, fait l'objet de peu de demandes directes de la part des retraités. Mais la petite taille des structures et leur absence de notoriété n'est peut-être pas le seul problème. Toutes les personnes qui y auraient intérêt s'orientent-elles vers le CCAS $?{ }^{17}$ Ou ne faut-il pas compter avec la réticence des personnes en difficulté à recourir aux institutions spécialisées dans le traitement des problèmes «sociaux»? La sollicitation de telles institutions est vécue comme un stigmate, et les personnes préfèrent pour cela se tourner vers des organismes plus «banals » comme la CPAM, même si ces institutions n'ont a priori aucune orientation généraliste :

préoccuper de la justice rendue aux usagers. Il propose une hypothèse selon laquelle le prestataire, face à l'usager, endosse des rôles, sur le registre d'un débat éthique qui déborde celui de l'application des règles formelles. Enfin, on retrouve derrière la sollicitude des agents le «régime de compassion» ou la «commune humanité » auxquels se réfèrent, respectivement, Philippe Corcuff (1996 [13]) et Nicolas Dodier (1997 [14]).

15. Cette sollicitude, qui trouve largement sa source chez les agents dans un «partage » des points de vue et de l'expérience même des usagers (Dodier, 1997 [14]), est à l'origine d'une lucidité remarquable des acteurs quant aux limites de leur propre secteur d'activité : «Moi j'ai maman qui a 80 ans aussi, elle a une aide ménagère aussi. C'est pas facile pour l'avoir, hein, c'est très très long, dis donc, il ne faut quand même pas être pressé... Bon ben c'est-à-dire qu'il faut faire un dossier assez important et puis après le temps que ça passe en commission, que ci, que là, moi en attendant... on a été obligé de payer quelqu'un d'autre...» (animatrice d'un centre pour personnes âgées).

16. Le point Infos Seniors a été créé à l'initiative d'un service chargé des relations avec les associations à caractère social de la mairie de Toulouse. La fondation de ce point d'information est partie d'un constat : le vieillissement des adhérents de clubs du troisième âge ainsi qu'une forte diminution de l'activité de ces clubs, et donc la nécessité de pallier la carence de ce type de structure en matière d'information des publics âgés. Le point info seniors a notamment pour objectif d'apporter des informations et des conseils dans différents domaines (juridique, assurance, organisation d'une animation, etc.) aux présidents de clubs souvent très âgés et peu entourés, et plus généralement de dynamiser l'univers des retraités et de proposer de nouveaux projets aux politiques. Le « point » répond aux demandes des personnes âgées en véhiculant des informations, en proposant des conseils sur les loisirs, les services que procure la mairie, les activités et organismes de Toulouse. Cette structure a pour prochain objectif de devenir un point central d'information pour les retraités ouverts aux associations et institutions qui pourraient y tenir des permanences.

17. Rappelons que le Centre Communal d'Action Sociale est une structure municipale chargée d'une action générale de prévention et de développement social dans la commune. En outre, le C.C.A.S. peut intervenir, sous forme de prestations remboursables ou non et de prestations en nature, afin de lutter contre la précarité, sans grever substantiellement les budgets locaux. La forme principale de l'action d'un C.C.A.S. reste la fourniture de secours en nature et en espèces afin de prévenir et de lutter contre toutes les formes d'exclusion sociale. Le C.C.A.S. peut gérer des services sociaux et médico-sociaux visant à la prévention et au développement social tels que : dispensaires, crèches, pouponnières, services d'aide ax personnes âgées et handicapées, foyersrestaurant et restaurants d'entraide, services d'aide ménagère et de soins infirmiers à domicile, ateliers d'assistance par le travail, etc. 
"L'AS, ils n'y vont pas parce qu'ils se sentent assistés, ils ont une certaine fierté, ils ont travaillé toute leur vie et ils viennent parce que, je crois moi que l'on est un peu anonyme tandis que AS ça fait plus assisté et ça leur fait plus peur. C'est le sentiment que j'en ai. » (CPAM).

Cette situation va à l'encontre de l'idée d'égalité d'accès aux services publics. L'égalité d'accès est le plus souvent posée en référence à l'usager et à ses caractéristiques sociographiques (selon une logique que résumerait assez bien un adage du type : «toutes les personnes qui remplissent les conditions ont droit à telle prestation»). Mais l'égalité d'accès aux services publics est rarement posée en référence au service public lui-même et à la façon dont il se présente ou «vient» aux usagers. De ce point de vue il convient de noter la différence qui s'établit entre certaines institutions (et/ou agents) qui optent d'emblée pour un tri efficace et un recentrage de leurs missions sur leur domaine de compétence strict...

"Parfois quand il s'agit de remplir des déclarations de ressources, on les aide à remplir leurs papiers. Mais enfin on essaie de minimiser ce genre de choses. Chacun sa mission. On n'est pas des écrivains publics, sinon on serait submergé de travail. D'ailleurs il n'y a pas assez d'écrivains publics. Il en faudrait des milliers. Pourquoi il n'y en en a pas plus? Je pense que c'est avant tout pour une raison économique. Vous savez ça coûte cher de créer des postes. » $(A S C A F)^{18}$.

... et les institutions qui acceptent une diversification de leur activité, au risque d'offrir des services quasi-clandestins, dont la connaissance ne se révèlera que dans l'interaction, à ceux des usagers qui seront assez malins, aventureux, mal informés ou naïfs pour faire l'expérience (réussie !) consistant à demander à l'institution ce qu'elle n'a pas à offrir :

«Ils viennent seuls avec leurs papiers, ils disent qu'ils ont oublié leurs lunettes, il y en a quelques-uns uns qui nous disent qu'ils ne savent pas lire. Ils viennent beaucoup pour qu'on leur remplisse leurs papiers. Ils viennent un peu moins maintenant parce que la pharmacie remplit avec l'ordinateur les feuilles de maladie mais c'est vrai qu'ils venaient pour ça. Mais il y a beaucoup de papiers pour lesquels ils sont obligés de venir. Alors ils viennent pour tout. Ils viennent pour la déclaration d'impôts, tout. Ils trouvent qu'ici on reçoit vite. »(CPAM).

18. Notons toutefois que ce genre de prise de position ne signifie nullement une fermeture de la personne sur une logique administrative au détriment d'une sensibilité «sociale ». Au contraire, la personne poursuit son propos en détaillant longuement le problème que pose l'illettrisme, l'impuissance et la révolte que lui suggère ce fléau. L'attitude de « recentrage sur la mission » relève donc moins d'une absence de sensibilité individuelle à la dimension «sociale élargie» des situations ( $c f$. le souhait de voir se créer des «milliers »d'emplois d'écrivains publics, qui vaut comme reconnaissance des problèmes à résoudre), mais des contraintes fonctionnelles qui pèsent sur les grands organismes, et qui imposent de limiter l'effort que l'on peut consentir aux situations atypiques pour assurer la «mission de base» de l'institution ( $c f$. le raisonnement quantitatif en termes de « postes », qui révèle la logique à l'œuvre dans ce genre d'institution). 
Les «assistantes sociales» sont ainsi redoublées ou secondées dans leur mission d'accompagnement des dossiers par d'autres acteurs : tout en signalant que ce n'est pas leur mission prioritaire, les Petits frères des pauvres, l'Atelier aîné Croix Rouge ou les membres de l'association Vivre à domicile reconnaissent prendre en charge la rédaction de certains courriers pour obtenir des aides sociales, et assistent les personnes dans la constitution de leurs dossiers ; l'écrivain public, loin de limiter son intervention aux questions de forme, tente de faire son métier d' «assistante sociale bis » ( $c f$. infra) en intervenant sur le fond, en gérant l'urgence, en décrochant son téléphone, en appelant les avocats, le notaire, la CRAM ou les ASSEDIC, en mobilisant toute l'expérience accumulée dans la gestion des détresses sociales auprès des divers organismes pour imaginer des solutions et faire avancer les dossiers, etc.

Parce que les agents des services sociaux doivent sans cesse arbitrer entre respect des règles et solution des cas individuels, et s'appuyer pour cela sur la formation qu'ils ont reçue et sur leur expérience des situations rencontrées, et parce que ces formations et ces expériences sont éminemment variables, ces professionnels sont inévitablement conduits, pour être efficaces et rendre un «bon» service aux personnes, à produire leurs propres «façons de faire » et leur propre arbitrage/arbitraire entre mission institutionnelle et assistance personnalisée. Ces formes d'arbitrages propres à l'agent sont la meilleure chose que ce dernier puisse faire et pourtant, leur juxtaposition au sein de mêmes organismes peut dérouter le public (au sens propre comme au sens figuré) et créer une certaine inégalité de traitement.

\section{B. Le paradoxe de la relation de service, ou la nécessaire autonomie des personnes que l'on souhaite assister}

Au paradoxe d'un problème social dont la résolution pose d'autres problèmes s'ajoute le paradoxe d'une représentation des personnes âgées comme «autonomes-dépendantes » nourrie par la pratique d'une relation de service contradictoire qui suppose un vis-à-vis doté d'une autonomie minimale et pourtant largement incapable de faire les gestes qui lui sont demandés. Tous les acteurs rencontrés s'accordent en effet pour valoriser l'autonomie, l'indépendance, l'activité plutôt que la passivité des personnes âgées... 19

19. On retrouve, derrière cette observation, les dilemmes de l'introduction volontariste de l'orientation client tels que les ont si bien identifiés Danielle Kaisergruber et Pierre Strobel (1996 [25]). Selon ces auteurs en effet, cette nouvelle préoccupation «a débouché sur la mise en place de technologies de communication entre les services publics sociaux et leurs usagers, et ce, dans une double perspective : faciliter l'accès de 
"Si je me mets à servir beaucoup, ils seront vite inefficaces, ils seront tributaires »; "Il faut qu'ils s'ouvrent vers les réseaux d'équipement social»; "ils ont de l'expérience et du savoir, il faut les mettre en valeur, ils n'étaient pas conscients du savoir qu'ils possédaient »

... mais pourtant, les mêmes acteurs sont conscients des limites de leurs propres souhaits : ils reconnaissent que les personnes sont mal informées, que les démarches administratives sont beaucoup trop complexes (on évoque «le parcours du combattant»), de sorte qu' «elles sont orientées plusieurs fois et finissent par abandonner». Selon certains agents, «[les personnes âgées sont perdues dans cet univers », «c'est tout un monde pour elles»; ces personnes manquent d'information, «ont du mal à comprendre comment fonctionne la société », ont tendance à « oublier », de sorte qu'il faut parfois noter pour elles sur un papier les démarches à effectuer. À l'extrême, les intervenants signalent la présence, même marginale, de personnes âgées qui n'ont plus de repères, et sur lesquelles il leur est par conséquent très difficile d'avoir prise : «elles sont dans la rue depuis longtemps, ont beaucoup de mal à se réinsérer quand les opportunités se présentent; il y a un travail à faire avant pour les réintégrer »; si ces personnes sont placées directement dans un établissement donné avec un cadre, des conditions, des règles, « elles sont en total décalage ». Après plusieurs démarches effectuées pour avoir droit à un logement certaines personnes sont au dernier instant reparties dans la rue, « on ne peut pas aller les chercher ».

La combinaison entre les récriminations exprimées plus haut quant à la «passivité » et quant à la demande d'assistance toujours accrue des personnes âgées, et la lucidité que nous découvrons quant à la fragilité des situations et quant à l'impuissance des institutions à leur venir en aide, montre les tensions dans lesquelles sont pris les personnels de l'aide sociale. Nombre d'entre eux s'efforcent de surmonter cette tension en nouant des contacts, des relations,

ces derniers à l'information et dans la mesure du possible promouvoir une logique du self-service, c'est-àdire de transfert d'une partie de la charge du travail vers les usagers (...). Loin d'intervenir en substitution des canaux plus traditionnels (courrier, visite aux guichets), cette offre nouvelle a généré sa propre demande fort inflationniste. Les nouveaux canaux eux-mêmes ont été vite pris d'assaut (notamment le téléphone); à tel point que leur saturation a dans certains cas contribué, par déversement, à engorger également les modes de contacts classiques. De plus, ces technologies sont plus ou moins - selon leur degré de sophistication discriminante. Si elles facilitent la vie aux usagers qui les manient aisément, en leur permettant de gérer à distance leur relation avec le service public, elles opèrent un filtrage sélectif, qui renvoie vers l'accueil physique, vers le guichet concret, les usagers les plus défavorisés. En même temps l'extension considérable de la pauvreté, la multiplication de situations sociales et familiales compliquées, de cas d'urgence ou de détresse majeure envoient aux mêmes guichets un nombre croissant de personnes, chacune porteuse d'un " cas " particulier ou urgent.» 
des partenariats et des collaborations «dans le dos» de ces usagers dont chacun sait bien qu'ils ne peuvent pas régler leurs problèmes par eux-mêmes. Le bon équilibre entre assistance et autonomie passe alors par une bonne gestion du problème de la dépendance.

\section{Travail des agents et relations entre services : personnalisation, complémentarité, concurrence}

\section{A. L'impossible personnalisation de l'offre de services : le cas de la couverture maladie universelle}

La gestion des problèmes est d'abord individuelle, comme le montre bien l'exemple de la difficile conciliation entre la Couverture Maladie Universelle et les attentes du public. Comme tout cadre national, la CMU se présente sous la forme d'une grille dotée de «seuils » ou de « trous » dans lesquelles ne rentrent pas toutes les situations particulières, d'où le stress que représente pour l'agent la nécessité de concilier respect du cadre et prise en compte des « cas » rencontrés :

"Maintenant il y a beaucoup de personne qui ne peuvent pas se payer de mutuelle avec cette CMU de base, personnes âgées ou pas. Mais vous avez par exemple des personnes qui ont le minimum vieillesse qui était je crois à $3540 \mathrm{~F}$. La CMU le niveau c'est $3400 \mathrm{~F}$. Donc $3540 \mathrm{~F}$...on revoit pour ces personnes là. Je pense qu'ils vont tout de même revoir ça. Là c'est national, nous on applique. »(CPAM).

Dans ce témoignage s'expriment la difficulté mais aussi le confort que représentent, pour la personne, le nécessaire respect d'un cadre sur lequel elle n'a aucune prise (« là c'est national, nous on applique »), et le sentiment de malaise personnel qui naît de la découverte quotidienne de l'absurdité du cadre ${ }^{20}$ — qui oblige les bénéficiaires du minimum vieillesse à souscrire, pour un dépassement de 140 francs, une mutuelle qu'ils n'ont manifestement pas les moyens de financer: comment expliquer à des personnes dont le minimum vieillesse vaut comme

20. Michael Lipsky (1982 [28]) avait observé des tensions analogues, en montrant que pour l'agent, prendre la défense de l'usager consiste parfois à entrer en conflit avec l'organisation. Cette observation conduisait l'auteur à poser le problème de l'aliénation en montrant les difficultés auxquelles s'exposent les agents quand ils doivent dénier l'humanité fondamentale qu'ils souhaiteraient témoigner envers les usagers pour effectuer leur travail dans les règles. De façon similaire, Jean-Marc Weller (1999 [39]) expliquait que le problème des agents consiste à pouvoir aligner des textes de droit, des principes de gestion et des requêtes civiles. La conséquence en est le «trouble du sens de l'intervention administrative ». Le caractère discrétionnaire des pratiques rendues nécessaires pour s'ajuster aux demandes des usagers afin de rendre l'action publique plus intelligente et plus responsable pose le problème de l'égalité de traitement. 
reconnaissance de leur précarité qu'elles n'ont pas droit à une couverture maladie destinée aux précaires?

«On ne sait pas comment solutionner tout cela, on ne le sait pas, et on n'a pas la capacité financière de leur payer une mutuelle, je ne sais pas. Je n'ai rien à leur proposer, on n'a rien à leur proposer. C'est depuis le $1^{e r}$ janvier que nous rencontrons ce problème. Puisque la CMU existe depuis le Ir janvier [2000].» (CPAM).

L'alternance du «je » et du «on» montre le délicat équilibre que tout agent d'organisme social doit sans cesse établir entre l'inévitable implication personnelle qu'il ressent face aux situations rencontrées au guichet et le non moins nécessaire respect du cadre légal qui fonde sa professionnalité.

Pour le dire en d'autres termes il y a sans nul doute, en arrière du marché de l'aide sociale, un «marché de l'altruisme », indissociable du caractère humain des prestations, et qu'on aurait tort de négliger : les cadres nationaux ont bien du mal à contenir la charge émotionnelle, la dimension affective, l'implication constante et sincère des acteurs du milieu, qui « prennent sur eux » une partie des détresses rencontrées. Face à cette difficulté les agents peuvent ou bien, comme nous le voyons ici, se retrancher derrière la règle en faisant le pari plus ou moins optimiste que les «responsables» prendront la mesure des difficultés et introduiront les réformes nécessaires («Je pense qu'ils vont tout de même revoir ça »), ou bien faire pression sur leur hiérarchie s'il s'agit de grands organismes ${ }^{21}$, ou se lancer eux-mêmes dans une extension de leurs prestations s'ils sont plus indépendants et bénéficient de marges de manœuvre.

L'impossible gestion individuelle de la relation de service se transpose alors au niveau collectif : comme nous allons le voir, les initiatives personnelles des agents contribuent à la prolifération et au brouillage des offres de service, au terme d'une surenchère vertueuse dans ses intentions, mais perverse dans ses effets : en voulant faire plus, en voulant faire mieux et contribuer à leur manière à un service plus proche des usagers, les acteurs de l'aide sociale se font concurrence, empiètent sur les domaines de compétences de leurs voisins, et contribuent de

21. L'étude sur laquelle se fonde notre analyse trouve son origine dans la prise en compte, par un grand organisme social, des «nouvelles demandes » qui se seraient exprimées au guichet, et dans le souci de la direction de prendre en compte la préoccupation de ses agents d'accueil soucieux de «faire quelque chose », mais ne sachant comment répondre aux attentes d'un public dont les difficultés personnelles débordent les missions strictes de l'institution. 
ce fait à complexifier la «lisibilité » des intervenants pourtant si nécessaire à la bonne orientation et prise en compte des publics concernés.

\section{B. La très fragile complémentarité entre offres de services : un trop plein et un manque de moyens}

Lorsqu'elles expriment les difficultés qu'elles rencontrent dans le cadre de leur activité, les personnes interrogées citent généralement deux éléments : le nombre élevé de dispositifs et la rareté des ressources, ce qui revient à exprimer la coexistence paradoxale d'un manque et d'un trop plein de moyens. Manque, dans la mesure où les ressources sont toujours insuffisantes pour couvrir l'ensemble des besoins : la récurrence des situations de détresse et l'aveu réitéré d'impuissance des agents de services publics face à nombre de ces situations suffisent à en témoigner. Trop plein, dans la mesure où la multiplicité des acteurs et le chevauchement des prestations crée comme nous l'avons vu des redondances, et débouche sur un gaspillage de ressources qui n'est pas seulement financier, mais cognitif, et dont il convient de souligner qu'il ne pénalise pas seulement les usagers (la demande), mais aussi les professionnels (l'offre); dans la mesure aussi où ces derniers ont du mal à s'y retrouver, et doivent déployer des efforts considérables pour identifier les bons interlocuteurs et nouer des coopérations efficaces pour l'avancement des dossiers :

"Il faut qu'on fasse appel directement aux organismes, il faut les trouver ces organismes, les personnes âgées des fois ne savent pas d'où ça vient, ne sait pas quelle est sa retraite, combien elle en a, enfin bon. » (AS CCAS)

"Les gens ne savent pas à qui s'adresser, et pour les assistantes sociales, ce n'est pas simple, il y a les assistantes sociales du secteur, qui travaillent pour les personnes âgées, il y a celles du Conseil Général. Il y a trois groupes que l'on a relevés, c'est le Conseil Général, la Mairie de Toulouse avec l'assistante sociale du CCAS et puis il y a la CRAM».

Il semble enfin que la mise en œuvre de telles collaborations et complémentarités repose pour une large part sur l'expérience individuelle des personnes :

"Ça fait neuf ans que je fais ce travail effectivement, bon, pas dans toutes les institutions, et puis les personnes changent aussi : il y a des personnes avec qui j'ai travaillé énormément d'années et qui n'y sont plus aujourd'hui, donc c'est d'autres personnes. Mais il faudrait à chaque fois capter les interlocuteurs, on ne peut pas faire autrement, généralement ça se passe très bien, j'ai les renseignements qu'il me faut, je renseigne la personne, jusqu'à présent ça fonctionne très bien. » (Écrivain public) 
Ce témoignage montre à quel point la configuration du réseau d'aide est dynamique, et répond davantage aux aléas des situations rencontrées, aux problèmes urgents, qu'à la mise en œuvre d'une architecture générale. C'est la force et la faiblesse du réseau. Force, dans la mesure où l'on puise dans cette organisation «itérative et spontanée » les moyens d'adapter rapidement les compétences de chacun à la résolution des problèmes rencontrés, et donc de pallier rapidement l'impuissance chronique des bureaucraties à faire entrer le particulier dans les cadres généraux (« ça se passe très bien, j'ai les renseignements qu'il me faut »). Faiblesse, dans la mesure où un tel processus détermine des « arrangements » très contingents, éphémères, mal connus, dépendant très largement de l'identité des personnes, des moments, des rencontres («il y a des personnes avec qui j’ai travaillé énormément d'années et qui n’y sont plus aujourd'hui »).

Le caractère pour une large part bricolé des arrangements entre acteurs du réseau montre la grande marge de manœuvre dont disposent les acteurs, et donc la fragilité des solutions coopératives. Cette fragilité désigne sa limite, à savoir l'existence, à côté des arrangements coopératifs, de situations où s'expriment plutôt la différenciation, la concurrence et le conflit, sans d'ailleurs qu'une configuration exclue l'autre : aussi paradoxal que cela puisse paraître, ce sont souvent les mêmes acteurs qui jouent simultanément sur les registres de la bonne volonté coopérative et d'une concurrence où s'expriment des intérêts plus étroits.

\section{Différenciation, concurrence et conflit}

L'exemple de l'écrivain public caractérise de façon emblématique les rapports de type concurrentiel qui s'expriment entre acteurs. Cette personne déclare avoir l'impression de faire "l'assistante sociale bis», tout en ajoutant que «ça n'est pas à l'écrivain public de faire ce genre de prestation». Ce discours est sincère. Il traduit à la fois un agacement devant les incohérences et les confusions qu'introduit la pluralité des intervenants, le souci de valoriser sa propre compétence, et le débordement plus général dont souffre chacune des personnes concernées. L'écrivain public refuse rarement, malgré une charge écrasante, d'aider les personnes qui viennent la voir, et pourtant ne nous cache pas qu'elle a l'impression de faire le travail d'autres organismes qui lui envoient des personnes. Elle a du mal à leur dire «écoutez non, je ne peux pas vous recevoir, allez voir la CRAM»(Écrivain public).

Cette situation est en fait généralisable aux autres acteurs en raison du fonctionnement particulier du marché de l'aide sociale, qui repose en quelque sorte sur un mécanisme de 
superposition et de délégation clandestines des interventions : d'un côté, dans un souci louable, mais souvent dicté par les aléas des situations rencontrées, le temps et les compétences dont on dispose, et/ou l'expérience personnelle que l'on a de ce genre de situations, les intervenants prennent en charge des problèmes qui ne sont pas «officiellement de leur ressort» et/ou « aiguillent » les personnes vers d'autres points du réseau, de sorte que chacun déborde sur l'action des autres et s'en plaint. Mais d'un autre côté, les mêmes acteurs s'appuient sur ce genre de débordement pour se valoriser.

Lorsque l'écrivain public se définit comme une «assistante sociale bis », le «bis » vise à souligner qu'elle n'est pas vraiment une assistante sociale, qu'elle prend en charge des activités qu'elle pourrait refuser, mais il signifie aussi que la personne ressent une certaine fierté d'exercer des compétences qui vont au-delà de ses attributions statutaires ; mieux : le « bis » signifie aussi et surtout que la personne accepte délibérément et volontiers de jouer sur ce registre, de faire mieux et clandestinement ce que les autres feraient officiellement mais moins bien. L'écrivain public se présente donc implicitement comme «concurrente » des assistantes sociales institutionnelles, et n'hésite pas à faire valoir ses atouts, ses «avantages concurrentiels »: réception «sans rendez-vous », prise en charge directe des demandes, disponibilité accrue à l'écoute et à l'intervention.

Chez l'écrivain public, le recours au registre de la différenciation «marchande » est très explicite : non seulement la personne qualifie spontanément sa disponibilité «d'atout de taille» (tout en déplorant le temps qu'une telle sollicitude lui coûte), mais elle recourt volontiers au registre de la comparaison quasi « consumériste » entre services concurrents, par exemple lorsqu'elle fait le parallèle avec l'interaction qui a lieu au guichet d'une petite banque, où des liens s’instaurent car les personnes se connaissent et se font confiance (« on avait le temps de discuter avec eux »), en stigmatisant par contraste les situations où l'accueil est plus distant, moins immédiat et plus anonyme :

"Dés l'instant que vous allez dans un bureau, c'est plus difficile, d'autant plus que tout est informatisé aujourd'hui (...) Ce qui revient c'est que les administrations ne réservent pas assez de temps aux personnes âgées donc pouvoir comprendre ce qu'ils veulent, mais je comprends aussi les administrations que quand ils ont une queue de trente personnes ils ne peuvent pas tout gérer. »(Écrivain public)

Le registre de la différenciation marchande n'oppose d'ailleurs pas uniquement les acteurs «périphériques » aux grandes structures, mais aussi les prestataires de même nature entre eux : 
"Déjà par rapport à l'[Association X], qui est une association d'aide ménagère, on n'est pas mal sur Toulouse, c'est quand même une grande association. Déjà donc elles [les aides ménagères] vont dans le domicile des personnes, donc par rapport à l'autonomie de la personne, quand elle baisse, quand les familles cherchent ou que la personne cherche un lieu de vie pour la fin de ses jours, quoi. Là, elles nous font de la publicité. »(Association Y)

Le discours relève bien du registre du «benchmarking», de la saisie des avantages comparatifs («par rapport à l'[Association X] [...] on n'est pas mal sur Toulouse»), des techniques de promotion marchande («elles nous font de la publicité »). Le réseau se construit en référence au marché : on se met en réseau pour s'abstraire de la concurrence.

Enfin, à l'attitude des acteurs qui voient dans les insuffisances supposées des grosses structures ou dans l'observation des voisins un moyen positif de trouver sa place et de valoriser sa différence, s'oppose la différenciation plus défensive des grands organismes, qui entendent rappeler des frontières trop souvent débordées par la diversité et l'inadéquation des demandes. Dans ce cas, la figure récurrente de l' «assistante sociale bis » fait l'objet d'un usage plus négatif, comme le montre ce témoignage d'un agent de la CPAM :

"Parce qu'en plus les assistantes sociales se servent de nous... on serait des assistantes sociales bis, donc je crois que c'est aux associations de bénévoles de remplacer ce pour quoi on a déjà suffisamment payé d'impôts, et l'assistante sociale doit faire son travail c'est sûr. »(CPAM)

Il s'agit ici d'attitudes très présentes dans les discours, et donc au-delà, dans les actes : d'un côté, les associations ou organismes, même s'ils ne sont pas qualifiés pour répondre à la demande des personnes, les aiguillent toujours, et ne les laissent ainsi jamais totalement démunies. Mais d'un autre côté, la nécessité permanente de ce combat pour les frontières et la récurrence de cet effort pour le réaiguillage montrent la complexité des problèmes, la fragilité des découpages sectoriels, et la nécessité, sinon d'acteurs et d'institutions «généralistes », du moins de médiateurs susceptibles à la fois de clarifier l'offre de service et d'accompagner les personnes dans leurs démarches :

"C'est les familles qui s'aperçoivent que leurs parents ne peuvent plus gérer, et ils nous alertent pour les repas, et nous on leur parle de la télé-assistance, donc on les oriente, on leur parle des numéros de téléphone qu'ils peuvent obtenir auprès du pôle social du CCAS, pour avoir le passage d'une assistante sociale pour évaluer les besoins (...). On a un coût des repas qui est assez élevé, et on a des personnes qui nous appellent parce que ça leur fait cher, donc ils nous alertent, donc on les oriente vers le Conseil Général puisque quand les personnes ont des revenus très modestes, elles peuvent bénéficier de la gratuité des repas (...) ». (Service portage de repas à domicile, CCAS) 
Ainsi on note un suivi au sein même des structures et une volonté de satisfaire chacun, attitude très fréquente chez les assistantes sociales :

«Il y a toujours un accueil téléphonique que nous faisons tous les jours, on tourne... Au niveau de l'écoute téléphonique, on fait une première évaluation, on essaie de comprendre la demande pour voir un petit peu la personne de quel organisme elle fait partie au niveau de sa caisse de retraite. Et à ce moment soit on conseille, soit on oriente, on guide déjà dès le premier appel téléphonique et puis tout dépend de la demande. » (AS CCAS)

Notons toutefois que le réaiguillage ne se suffit pas à lui-même : tous ces exemples nous montrent que la réorientation des personnes suppose souvent d'une part l'intervention de leur entourage, et d'autre part la participation active de ces personnes elles-mêmes. Ces deux conditions pointent un double problème : la première souligne, par contraste, le handicap terrible que représente l'isolement des personnes âgées, un problème que les institutions ne peuvent pallier à elles seules ; la seconde illustre le pis-aller que représente le réaiguillage par rapport à la découverte immédiate de la bonne porte ou l'accompagnement physique des personnes.

\section{Conclusion}

Le secteur de l'aide sociale se présente bien, même à l'insu des acteurs, comme un marché, puisqu'il fait référence à une relation de service conçue implicitement comme la rencontre d'une offre et d'une demande, avec des guichets qui fonctionnent comme des boutiques, c'est-àdire qui mettent en jeu des prestations spécialisées dûment signalées et codifiées, des prestataires attentionnés, la possibilité d'un échange et d'une négociation, même s'il est vrai qu'ici la notion de prix n'intervient pas (sauf peut-être de façon indirecte, en termes de temps passé d'un côté et de l'autre, et d'investissements et de financements publics du côté des prestataires). Mais en face, les personnes ne savent pas forcément percevoir et présenter leurs problèmes comme une «demande » qu'il s'agit à la fois d'orienter, par le calcul, vers l'offre adéquate, et ensuite d'exprimer et d'argumenter comme il convient auprès des représentants de l'«offre $»^{22}$. En d'autres termes, s'il y a bien un marché de l'aide sociale, la question qui se

22. En même temps cette demande n'apparaît jamais neutre aux yeux des agents, elle fait toujours l'objet d'une interprétation qui rend compte du service rendu. Philippe Warin (1993 [35]) a ainsi montré combien la reconnaissance des conduites des usagers est conditionnée par une combinaison de contraintes de recevabilité vis-à-vis du système de valeur des agents, vis-à-vis des schémas d'interprétation disponibles dans le service, vis-à-vis des stratégies singulières des agents ou des organes administratifs. La compétence interprétative des 
pose est de savoir s'il s'agit d'un marché achevé, s'il y a vraiment des consommateurs rationnels et calculateurs sur ce marché. La situation marchande qui règne du côté de l'offre ne se retrouve pas du côté de la demande... un terme qui caractérise assez mal la situation et les dispositions du public concerné. Or cette asymétrie, cette incomplétude du modèle, est précisément à l'origine de l'ensemble des problèmes que nous avons constatés.

Pour le dire plus précisément, il faudrait se garder de postuler comme on a trop souvent tendance à le faire que l' « usager » est le frère jumeau du « citoyen » ou du « consommateur », comme si les personnes étaient toujours capables d'identifier leurs souffrances et leurs sentiments à des «besoins », de rapporter leurs difficultés à des «droits », et surtout de les orienter spontanément et rationnellement vers les institutions ad hoc (c'est-à-dire de faire « usage » et «bon usage» des services publics) $)^{23}$. Notre étude montre plutôt que la notion d' «aide sociale» est assez peu compatible avec la prise en compte d'une demande : si les personnes ont besoin d'être aidées, ce n'est pas seulement pour répondre à leurs questions et à leurs sollicitations directes, mais encore et surtout pour leur permettre de prendre conscience de leurs difficultés, et de découvrir l'existence de dispositifs propres à les résoudre. L'aide sociale consiste non seulement à «attendre » et à « résoudre » les problèmes personnels, mais aussi et plus fondamentalement à aller au-delà de cette relation de marché en créant paradoxalement les conditions favorables à son expression, par exemple en intervenant de façon volontaire auprès des personnes pour les aider à formuler leur vécu sous forme de questions et de sollicitations ${ }^{24}$.

agents a également été soulignée par John Gumpertz (1995 [22]) à partir d'un examen minutieux des activités langagières déployées lors d'entretiens de sélection pour des stages de formation. L'auteur montre que cette activité interprétative des agents n'est pas sans effets pervers, dans la mesure où les usagers dont les pratiques communicatives ne correspondent pas au modèle de conversation communément partagé rencontrent beaucoup plus de difficulté que les autres dans leurs contacts institutionnels.

23. Lipsky (1982 [28]) montre combien la relation de service repose sur le «mythe de l'altruisme ». Les usagers, loin d'attendre que les agents se comportent en simples gardiens passifs accordant ou refusant l'accès aux demandes selon des critères définis à l'avance sont plutôt enclins à penser que ces mêmes agents se feront leurs avocats, et mobiliseront donc leur savoir, leurs compétences et leur position pour leur assurer le meilleur traitement ou la meilleure position compatible avec les contraintes du service. En d'autres termes, les usagers sont portés à faire confiance à des étrangers et à se laisser manipuler et commander dans l'attente d'une aide et d'un traitement justes.

24. On retrouve ici les enseignements d'une étude sur l'interaction au guichet dans les caisses d'allocations familiales, qui montrait bien que les allocataires étaient en quête d'écoute plus que d'efficacité (Dubois, 1996 [15]). 
De ce point de vue et comme nous l'avons souligné, la prolifération des intervenants et surtout l'absence de réflexion des acteurs et des chercheurs sur cette prolifération posent problème. Si l'on dresse la liste non seulement des quelques acteurs que nous avons interrogés à Toulouse, mais aussi des autres intervenants cités par ces acteurs, on s'aperçoit qu'une centaine d'institutions, organismes et autres associations ont été mis en avant... et l'on peut supposer que cette énumération est loin d'être exhaustive. On assiste ainsi à une multiplication des services et des structures qui diversifie, complète, voire double celles qui existent déjà. Les relations qu'entretiennent cette série d'intervenants vont de l'ignorance réciproque à la coopération, en passant par la subordination, la complémentarité, et la concurrence... Désormais, de nombreuses structures travaillent dans le même sens, tout en proposant des prestations identiques; une dimension de rentabilité a même parfois été introduite. C'est pourquoi certains n'hésitent pas à reprendre l'idée de «marché » au sens économique du terme :

«Oui, c'est un marché. Vous n'avez qu'à voir comment se passent les voyages $d u$ troisième âge. La crainte que j'ai, moi, personnellement, c'est que le secteur marchand arrive sur les aides à domicile et qu'il n'y ait plus d'associations 1901, mais un secteur marchand qui va casser tous les prix et va faire n'importe quoi. On le voit déjà, il y a des associations qui se montent, il n'y a aucune formation dans le social, c'est des ingénieurs en électronique. Ils n'ont jamais vu de personnes âgées, ils ne savent pas ce que c'est qu'une pathologie, et ils montent une association. Sur tous les secteurs c'est comme cela, après c'est à nous de nous battre pour faire reconnaître notre métier et apporter un service de qualité. C'est sûr qu'il va y avoir des gens qui vont en profiter, je crois, moi, qu'il faudra faire attention. Il y a des escrocs, faut voir! Et puis les personnes âgées sont quand même des gens vulnérables, et ça on ne peut rien y faire ». (Vice-Présidente d'une association).

Le secteur de l'aide sociale aux personnes âgées est un marché très particulier, où les clients ne sont pas ceux qu'on croit : il s'agit de capter un premier public proche (les vieux) pour capter des flux financiers distants ; on utilise un centre de décision vulnérable pour en mobiliser un autre. Ce témoignage confirme en outre que l'associatif n'est pas exclusif du marchand, dans la mesure où l'impossibilité d'un profit financier n'exclut pas des profits sociaux (Gadrey, 2000 [19]), en termes d'activité notamment (si créer une association ne peut avoir, par définition, de but lucratif, la même création est tout de même pourvoyeuse d'emplois, c'est-à-dire de richesses qui ne sauraient être négligées). L'intrusion croissante de cette nouvelle dimension du marché dans le monde de l'assistance aux personnes a pour résultat d'aboutir à une aide sociale «éclatée », « démembrée », difficilement lisible, où le meilleur côtoie le pire, d'où l'importance d'une réflexion globale sur le tri des intervenants, sur la 
coordination des partenaires fiables, et sur la visibilisation de ce «réseau» dans lequel l'usager se trouve souvent perdu et/ou peut se perdre.

\section{Références}

[1] BOLTANSKI L. \& THEVENOT L. (1991), De la justification, Les économies de la grandeur, Paris, Gallimard.

[2] BORZEIX A. (1995), «Qualité et bienveillance : l'épreuve de l'étranger », in Joseph I. \& Jeannot G., Les compétences de l'agent et l'espace de l'usager, Paris, CNRS éditions, pp. 87-123.

[3] CALLON, M. (1998), «Introduction: The Embeddedness of Economic Markets in Economics », in Callon M. (ed.), The Laws of the Markets, Oxford, Blackwell, pp. 2-57.

[4] CALlON M., MEADEL C. \& RABEHARISOA V. (2000), «L'économie des qualités », Politix vol.13, $\mathrm{n}^{\circ}$ 52, pp. 211-239.

[5] CHAUVIERE M. (1997), «Le champ familial : des usagers aux rapports sociaux d'usage », in Warin, Philippe, Quelle modernisation des services publics? Les usagers au cœur des réformes, Paris, La Découverte, pp. 221-242.

[6] CHAUVIERE M. (2000), «Usages et significations contradictoires de la "relation de service" dans le secteur social », in Rouban, Luc, Le service public en devenir, Paris, L'Harmattan, pp. 83-106.

[7] COCHOY F. (2001a), «Les effets d'un trop-plein de traçabilité », La Recherche, n ${ }^{\circ}$ spécial Le risque alimentaire, $\mathrm{n}^{\circ} 339$, février, pp. 66-68.

[8] COCHOY F. (2001b), La CRAM dans le marché de l'aide sociale, rapport de recherche rédigé pour la Caisse Régionale d'Assurance Maladie de la Haute-Garonne, en collaboration avec l'I.U.P.S.A., Université Toulouse II, mars (trois tomes).

[9] COCHOY F. (2001c) «La captation des publics entre dispositifs et dispositions, ou le petit chaperon rouge revisité. Pour une sociologie du travail relationnel », Journées d'étude sur les dispositifs de gestion, Université de Versailles Saint-Quentin en Yvelines, 4-5 octobre 2001.

[10] COCHOY F. (2002), Une sociologie du packaging, ou l'âne de Buridan face au marché, Paris, Presses Universitaires de France.

[11] COCHOY F. GAREL J.-P. et TERSSAC G. de (1998), «Comment l'écrit travaille l'organisation : le cas des normes ISO $9000 »$, Revue française de sociologie, décembre 1998, vol. 39, n 4, décembre, pp. 673-699.

[12] COCHOY F. \& TERSSAC G. de (1999), «Les enjeux organisationnels de la qualité : une mise en perspective », Sciences de la société, numéro spécial Organisation et qualité, n 46, février, pp. 3-18.

[13] CORCUFF Ph. (1996), « ordre institutionnel, fluidité situationnelle et compassion — Les interactions au guichet de deux CAF », Recherches et Prévisions, $\mathrm{n}^{\circ}$ 45, septembre.

[14] DODIER N. (1997), «Réseaux socio-techniques et conscience du collectif», Sociologie du travail, vol. $39, \mathrm{n}^{\circ} 2$, pp. 131-148.

[15] DUBOIS V. (1996), «Une institution redéfinie par ses usage(r)s ? Sur quelques pratiques du guichet dans les Caisses d'Allocations Familiales », Recherches et prévisions, $\mathrm{n}^{\circ}$ 45, septembre, Paris, CNAF, pp. 5-15. 
[16] FIJALKOW Y. (2002), «Les visages réversibles de l'usager et du consommateur : ce que nous apprend la grève des internautes », Sciences de la société (à paraître).

[17] FITOUSSI J.-P. et ROSANVALLON P. (1996), Le nouvel âge des inégalités, Paris, Seuil.

[18] GADREY J. (1994), «Les relations de service et l'analyse du travail des agents », Sociologie du travail, n 3, pp. 381-389.

[19] GADREY J. (2000), «Le tiers secteur comme objet d'étude : quel objet, quelles études ? », Sociologie du travail, vol. 42, n 4 , pp. 601-606.

[20] GAUTRAT J. (1996), «Les Caisses d'Allocations Familiales confrontées à l'entrée en masse de nouveaux usagers », in Grémion, Catherine, Fraisse, Robert., Le service public en recherche. Quelle modernisation? Paris, La documentation Française, pp. 241-256.

[21] GROSJEAN M. et LACOSTE M. (1998), «L'oral et l'écrit dans les communications de travail ou les illusions du tout écrit », Sociologie du travail, $\mathrm{n}^{\circ}$ 4, pp. 439-461.

[22] GUMPERZ J. (1995), «Les processus inférentiels dans les interactions de service », in Joseph, Isaac \& Jeannot, Gilles, Les compétences de l'agent et l'espace de l'usager, Paris CNRS éditions, pp. 41-62.

[23] JEANNOT G. (1999), «Services publics : l'usager, le client, le consommateur », Urbanisme, n 307, juillet août 1999, pp. 53-55.

[24] JEANTET A. (1998). «Les objets intermédiaires dans la conception. Éléments pour une sociologie des processus de conception », Sociologie du travail, vol. 40, n 3, pp. 291-316.

[25] KAISERGRUBER D. \& STROBEL P. (1996), « Service public et fin de siècle. Contraintes européennes et défi de la pauvreté», in Grémion C. \& Fraisse R., Le service public en recherche. Quelle modernisation? Paris, La documentation Française, pp. 21-51.

[26] KARPIK L. (1989), «L'économie de la qualité », Revue Française de Sociologie, vol. 30, n² 2, avril-juin, pp.187-210.

[27] LESEMANN F. et CHAUME C. (1992), «Quand usagers et producteurs de service ne se rencontrent pas... À propos d'une recherche sur le maintien à domicile des personnes âgées », in Chauvière, Michel et Godbout, Jacques T., Les usagers entre marché et citoyenneté, Paris, L'Harmattan, pp. 159-166.

[28] LIPSKY M. (1982), Street Level Bureaucracy: Dilemnas of the Individual in Public Services, New York, Russel Sage Foundation.

[29] NORMAN D. A. (1993), «Les artefacts cognitifs », Raisons Pratiques, Les objets dans l'action, Paris, Éditions de l'E.H.E.S.S., $n^{\circ}$ 4, pp. 15-34.

[30] PAUGAM S. (dir.) (1996), L'exclusion, l'état des savoirs, Paris, La Découverte.

[31] PIOTET F. (1997), «Les événements de décembre 1995, chronique d'un conflit », Sociologie du travail, $\mathrm{n}^{\circ} 4$, pp. 523-546.

[32] POLANYI K. (1983), La grande transformation, Paris, Gallimard.

[33] ROSANVALLON P. (1998), La nouvelle question sociale : repenser l'Etat providence, Paris, Seuil.

[34] STROBEL P. (1993), «L'usager, le client et le citoyen: quels rôles dans la modernisation du service public ?» Recherches et prévisions, $\mathrm{n}^{\circ} 32$, juin, pp. 31-44. 
[35] WARIN Ph. (1993), Les usagers dans l'évaluation des politiques publiques : études des relations de service, Paris, L'Harmattan.

[36] WARIN Ph. (1999), La performance publique : attentes des usagers et réponses des ministères. Revue politiques et management public, vol. $17, \mathrm{n}^{\circ} 2$, pp. 147-164.

[37] WARIN Ph. (2000), «Les relations de service : miroir des décalages entre politique de modernisation administrative et attentes sociale », in Rouban, Luc, Le service public en devenir, Paris, L'Harmattan, pp. 63-81.

[38] WELLER J.-M. (1998), «La modernisation des services publics par l'usager : une revue de littérature », Sociologie du travail, $\mathrm{n}^{\circ} 3$, pp. 365-392.

[39] WELLER J.-M. (1999), L'État au guichet, Paris, Desclée de Brouwer. 

Franck Cochoy est Professeur de sociologie à l'Université Toulouse II et membre du CERTOP (UMR CNRS 5044). Il consacre ses travaux à la sociologie du marché. Il est l'auteur de Une histoire du marketing, Paris, La Découverte, 1999 et de Une sociologie du packaging ou l'âne de Buridan face au marché, Paris, PUF, 2002. Ses principaux articles ont paru dans Les cahiers internationaux de sociologie, Genèses, Gérer et comprendre, Politix, Réseaux, la Revue française de gestion, la Revue française de sociologie, Sociologie du travail.

Ygal Fijalkow est ATER en sociologie à l'Université Toulouse et membre du CERTOP, où il prépare une thèse de doctorat intitulée Entre logique marchande et logique citoyenne : les conduites des usagers face à l'ouverture à la concurrence des entreprises publiques, sous la direction de Jean-Yves Nevers. Ses recherches portent sur les rapports qu'entretiennent les usagers au service public dans un contexte de déréglementation, d'ouverture à la concurrence, de privatisation et de marchandisation de l'offre de service. 